\title{
Thoratec left ventricular assist device as a bridge to recovery in a child weighing 27 kilograms
}

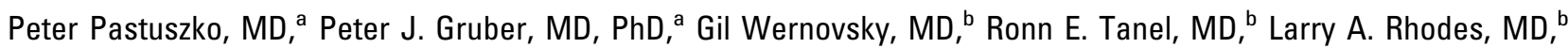 \\ Susan C. Nicolson, MD, ${ }^{c}$ Thomas L. Spray, MD, and J. William Gaynor, MD, ${ }^{a}$ Philadelphia, $\mathrm{Pa}$
}

Because of a potential conflict of interest related to this article on the part of our editors, Dr Richard Jonas served as guest section editor, assigned reviewers, and made editorial decisions or recommendations leading to its acceptance for publication.

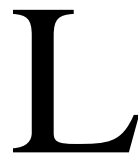

imited organ availability and prolonged waiting periods for donor hearts has led to the increased use of ventricular assist devices (VADs) as a bridge to transplantation. Recognition of potential myocardial recovery in some reversible cardiac diseases has prompted the use of these devices as a bridge to recovery. Extracorporeal membrane oxygenation is often the only choice for circulatory support in infants and smaller children. ${ }^{1}$ There are currently no pulsatile VADs approved for use in infants and children in the United States. ${ }^{2}$ Adult devices such as the Thoratec VAD (Pleasanton, Calif) have been used in some children as a bridge to transplantation. ${ }^{3-5}$ However, the discrepancy in size between the device and the patient limits its use, particularly if bridge to recovery and removal of the device are planned.

\section{Clinical Summary}

An 11-year-old previously healthy girl was noted by her grandmother to have an "irregular heartbeat" associated with gasping and agonal respirations while sleeping. Cardiopulmonary resuscitation was initiated, and emergency medical services were called. Ventricular fibrillation was detected and successfully cardioverted to sinus rhythm. After transport to a local hospital, the patient was transferred to The Children's Hospital of Philadelphia. Refractory polymorphic ventricular tachycardia resulted in hypotension and low cardiac output. A lidocaine infusion was begun with poor control of the ventricular ectopy. Echocardiography revealed mod-

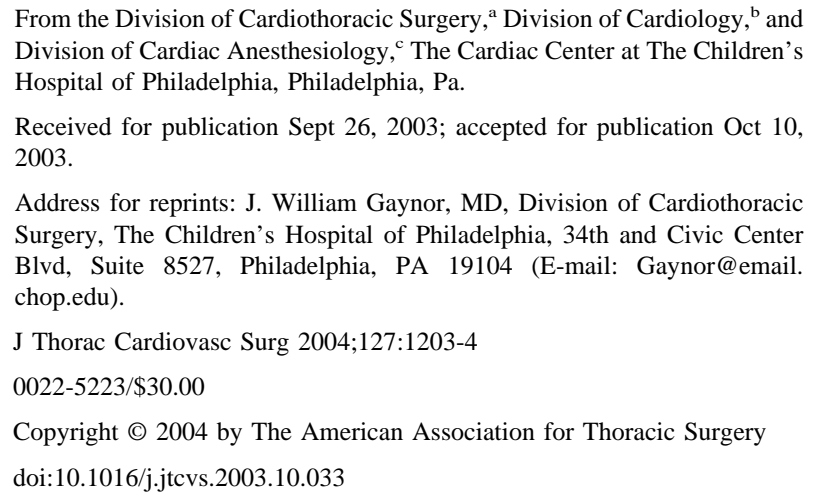

erate left ventricular dysfunction with a shortening fraction of $19 \%$ and moderate mitral regurgitation. Right ventricular function was preserved. Initiation of inotropic drug therapy resulted in increased ectopy and further deterioration. Because of the isolated left ventricular dysfunction, she was a candidate for left ventricular assist device (LVAD) support.

The patient was taken to the operating room for insertion of a Thoratec LVAD. After cardiopulmonary bypass was initiated, the inflow cannula was positioned in the ventricular apex. An alternative technique would be the use of atrial cannulation for VAD inflow; however, filling of the VAD may be improved with use of apical cannulation. The outflow graft $(14 \mathrm{~mm})$ was anastomosed to the ascending aorta. The conduits were de-aired and connected to the device. The patient was weaned from bypass with the device in the volume mode, but later it was converted to the fixed rate mode secondary to inadequate filling of the VAD. The early postoperative course was significant for right ventricular dysfunction requiring aggressive volume resuscitation and support with inotropic drug therapy to optimize preload to the VAD. Amiodarone therapy was initiated because of refractory ventricular ectopy. She was extubated on postoperative day 2. A heparin infusion was used for anticoagulation with no bleeding complications. Mild hemolysis was the only device-related morbidity. Serial echocardiograms demonstrated complete recovery of ventricular function.

The VAD was removed after 13 days of support. The patient was placed on cardiopulmonary bypass. The outflow cannula was stapled with a vascular stapler and divided at the aorta. The cannula was removed from the left ventricular apex, leaving the sewing ring, which was approximated using pledgetted 0 sutures. After the patient was separated from cardiopulmonary bypass, transesophageal echocardiography demonstrated normal ventricular function. Because of persistent ventricular ectopy, despite amiodarone therapy, she underwent implantation of a transvenous defibrillator (internal cardioverter-defibrillator). She was subsequently transitioned to mexiletine therapy. An echocardiogram performed 5 months after discharge demonstrated normal ventricular function. At that time, she had no cardiovascular symptoms. Recurrent ventricular fibrillation has been detected by the internal cardioverter-defibrillator. The cause of the polymorphic ventricular arrhythmia has not been determined.

\section{Discussion}

The largest experience with pulsatile VADs in children is in Europe. The Berlin Heart (Berlin, Germany) and the MEDOS (Stolberg, Germany) assist devices are available in a variety of sizes, are suitable for all age groups, and have been used successfully as bridge to recovery and transplant. Neither the Berlin Heart nor the MEDOS VAD are available in the United States, and no pulsatile device is currently approved for use in pediatric patients. 
The Thoratec VAD has been used in a small number of children according to the manufacturer's database. As of May 2003, the Thoratec VAD had been used as a bridge to transplant in the United States in 8 children weighing less than $30 \mathrm{~kg}$, but it had not been used in any children as a bridge to recovery. The smallest child was $17 \mathrm{~kg}$. ${ }^{5}$ Unlike extracorporeal membrane oxygenation, during the period of VAD support patients can be extubated and ambulate, allowing rehabilitation. There is no single solution for pediatric patients requiring mechanical support, but carefully selected pediatric patients may benefit from the Thoratec device as a bridge to transplant recovery. The current case demonstrates that, even in a relatively small child, the device can be removed without adverse effects on ventricular function despite apical cannulation.

\section{References}

1. Duncan B. Mechanical circulatory support for infants and children with cardiac disease. Ann Thorac Surg. 2002;73:1670-7.

2. Reinhartz O, Stiller B, Eilers R, Farrar DJ. Current clinical status of pulsatile pediatric circulatory support. ASAIO J. 2002;48:455-9.

3. Reinhartz O, Keith FM, El-Banayosy A, McBride LR, Robbins RC, Copeland JG, et al. Multicenter experience with the Thoratec ventricular assist device in children and adolescents. J Heart Lung Transplant. 2001;20:439-48.

4. Williams MR, Oz MC. Indications and patient selection for mechanical ventricular assistance. Ann Thorac Surg. 2001;71:S86-91.

5. Copeland JG, Arabia FA, Smith RG. Bridge to transplantation with a Thoratec left ventricular assist device in a $17 \mathrm{~kg}$ child. Ann Thorac Surg. 2001;71:1003-4.

\title{
The bovine jugular vein conduit for right ventricular outflow tract reconstruction: A feasible alternative to homograft conduits?
}

\author{
Tomaso Bottio, MD, PhD, ${ }^{a}$ Gaetano Thiene, MD, ${ }^{\mathrm{b}}$ Vladimiro Vida, MD, ${ }^{a}$ Mila Della Barbera, MD, ${ }^{\mathrm{b}}$ Annalisa Angelini, MD,
} Giovanni Stellin, MD, and Gino Gerosa, MD, ${ }^{a}$ Padua, Italy

T he bovine jugular vein conduit (BJVC) has been proposed for use in cardiac surgery for correction of congenital heart defects, either for right ventricular outflow tract (RVOT) reconstruction ${ }^{1}$ or total cavopulmonary anastomosis completion in Fontan circulation. ${ }^{2}$ Short-term and midterm results with the BJVC are controversial: satisfactory when used for RVOT reconstruction but rather disappointing for Fontan completion. In this study we report our early and late clinical results with BJVCs implanted in infants and children for RVOT reconstruction.

\section{Clinical Summary}

Between June 1999 and December 2002, 10 patients (7 male and 3 female patients) with a median age of 2 months (mean age, 13.7 \pm 25.1 months) underwent surgical repair of congenital heart defects, including reconstruction of the RVOT with a BJVC (Contegra; Medtronic, Inc, Minneapolis, Minn).

From the Departments of Cardiovascular Surgery ${ }^{\mathrm{a}}$ and Cardiovascular Pathology, ${ }^{\text {b }}$ University of Padua Medical School, Padua, Italy.

Supported by grants of Istituto Superiore di Sanità and Ministero Istruzione Universita' e Ricerca and by Regione Veneto, Venice, Italy.

Received for publication Aug 8, 2003; revisions received Sept 6, 2003; accepted for publication Sept 10, 2003.

Address for reprints: Tomaso Bottio, MD, Department of Cardiovascular Surgery, Via Giustiniani, 2, 35128 Padua, Italy (E-mail: tomaso.bottio@unipd.it).

J Thorac Cardiovasc Surg 2004;127:1204-7

$0022-5223 / \$ 30.00$

Copyright $\odot 2004$ by The American Association for Thoracic Surgery

doi:10.1016/j.jtcvs.2003.11.005
Patient demographics are detailed in Table $1 .^{3}$ Truncus arteriosus was the indication in 5 patients (with right ventricular hypoplasia in 1 patient), tetralogy of Fallot with pulmonary atresia in 2 patients, aortic arch interruption type B with severe aortic stenosis in 1 patient, double-outlet right ventricle with pulmonary atresia in 1 patient, and aortic stenosis in 1 patient. Four patients had undergone previous palliative procedures.

\section{Results}

There were 2 (20\%) hospital deaths. One patient (patient 3) operated on for truncus arteriosus died suddenly on the 52nd postoperative day at home. The second patient (patient 5), who underwent a Ross-Konno procedure and a mitral valvuloplasty for aortic and mitral stenosis, died on the first postoperative day after an airway suction procedure. A third patient (patient 8), a baby girl operated on for truncus arteriosus type A-4 of Van Praagh classification, died of respiratory distress syndrome on the 20th postoperative day after extracorporeal membrane oxygenator assistance. Postmortem examination was performed in patients 3 and 5 .

The remaining 7 patients were alive at the last follow-up. Echocardiographic data, recorded after a median time of 25 months (mean time, $24.3 \pm 14$ months), showed trivial incompetence of the BJVC in 3 patients and moderate incompetence in 1 patient. A transvalvular vein conduit peak gradient of greater than $30 \mathrm{~mm} \mathrm{Hg}$ was observed in 2 patients. In 1 patient a peak gradient of greater than $30 \mathrm{~mm} \mathrm{Hg}$ was detected at the level of the distal anastomosis between the conduit and the left pulmonary artery.

Morphologic investigation was performed in an unimplanted conduit that served as a control (Figure 1) and in the 2 conduits explanted at autopsy (Figure 2).

The unimplanted conduit showed a well-fixed tissue with leaflets consisting of a compact layer of collagen (fibrosa) and a layer 